\title{
Philosophiques
}

\section{L’égalité politique et les limites de la démocratie procédurale}

\section{Florent Guénard}

Volume 46, numéro 1, printemps 2019

La démocratie entre substance et procédure

URI : https://id.erudit.org/iderudit/1062011ar

DOI : https://doi.org/10.7202/1062011ar

Aller au sommaire du numéro

Éditeur(s)

Société de philosophie du Québec

ISSN

0316-2923 (imprimé)

1492-1391 (numérique)

Découvrir la revue

Citer cet article

Guénard, F. (2019). L'égalité politique et les limites de la démocratie procédurale. Philosophiques, 46(1), 29-44. https://doi.org/10.7202/1062011ar

\section{Résumé de l'article}

Tout le monde semble s'accorder pour penser la démocratie comme le régime de l'égalité politique. Mais les divergences sont nombreuses dès qu'on s'interroge sur le contenu de cette égalité et sur les raisons qu'on peut invoquer pour la justifier.

Deux types d'arguments semblent s'opposer sur ce point. On peut considérer, comme R. Dahl par exemple, que l'égalité politique est requise afin que la désignation des gouvernants par la voie électorale puisse paraître légitime et qu'elle ne soit pas contestée. L’argument est alors procédural. On peut également considérer, comme T. Nagel par exemple, que cette justification est insuffisante parce qu'elle est extérieure à l'idée démocratique, et que si celle-ci implique que tous aient le même statut politique, c'est parce qu'elle est le régime général de l'égalité, et que l'égalité politique en est la première forme. L'argument est alors substantiel.

L'objet de cet article est double. Il s'agit d'une part de comprendre ce que les défenseurs d'une conception substantielle de la démocratie reprochent à ceux qui définissent celle-ci en termes procéduraux. Il s'agit d'autre part de se demander s'il est possible de s'en tenir à une justification seulement procédurale de l'égalité politique, et en conséquence de s'interroger sur l'interprétation que l'on doit en avoir.
Ce document est protégé par la loi sur le droit d'auteur. L'utilisation des services d'Érudit (y compris la reproduction) est assujettie à sa politique d'utilisation que vous pouvez consulter en ligne.

https://apropos.erudit.org/fr/usagers/politique-dutilisation/ 


\title{
L'égalité politique et les limites de la démocratie procédurale
}

\author{
FLORENT GUÉNARD \\ Maître de conférences de philosophie à l'ENS UIm
}

\begin{abstract}
RÉSUMÉ. - Tout le monde semble s'accorder pour penser la démocratie comme le régime de l'égalité politique. Mais les divergences sont nombreuses dès qu'on s'interroge sur le contenu de cette égalité et sur les raisons qu'on peut invoquer pour la justifier.

Deux types d'arguments semblent s'opposer sur ce point. On peut considérer, comme R. Dahl par exemple, que l'égalité politique est requise afin que la désignation des gouvernants par la voie électorale puisse paraître légitime et qu'elle ne soit pas contestée. L'argument est alors procédural. On peut également considérer, comme T. Nagel par exemple, que cette justification est insuffisante parce qu'elle est extérieure à l'idée démocratique, et que si celle-ci implique que tous aient le même statut politique, c'est parce qu'elle est le régime général de l'égalité, et que l'égalité politique en est la première forme. L'argument est alors substantiel.

L'objet de cet article est double. Il s'agit d'une part de comprendre ce que les défenseurs d'une conception substantielle de la démocratie reprochent à ceux qui définissent celle-ci en termes procéduraux. Il s'agit d'autre part de se demander s'il est possible de s'en tenir à une justification seulement procédurale de l'égalité politique, et en conséquence de s'interroger sur l'interprétation que l'on doit en avoir.
\end{abstract}

ABSTRACT. - Democracy is usually seen as the regime of political equality. But there are many differences about the content of this equality and the reasons that can be invoked to justify it.

Two types of arguments seem to be opposed on this point. One can consider, as R. Dahl for example, that political equality is required so that the election of the leaders can appear legitimate and not disputed. The argument is then procedural. One can also consider, as T. Nagel for example, that this justification is insufficient because it is external to the democratic idea, and that if this implies that all have the same political status, it is because democracy is the general system of equality, and political equality is its first form. The argument is then substantial.

The aim of this article is twofold. First, I try to understand why the defenders of a substantial conception of democracy blame those who define it in procedural terms. Next, I try to understand the reason why the procedural justification of political equality could be irrelevant, and must be completed by substantial arguments.

La conception que nous nous faisons de la démocratie est généralement déterminée par deux attentes à l'égard de ce type de régime. Nous attendons que la démocratie soit le système politique qui associe chacun à la décision publique. Mais nous souhaitons également que la démocratie mette en place des politiques en vue du bien commun, conduites de telle sorte que chaque 
individu fasse, dans la mesure du possible, l'objet d'un traitement égal. Ces deux attentes, on le sait, ne sont pas immédiatement compatibles. Nous sommes intimement persuadés qu'une politique discriminatoire ne peut pas être qualifiée de démocratique; mais nous sommes également intimement persuadés qu'il n'est pas totalement légitime de restreindre l'égalité politique au nom d'une politique du bien commun. La démocratie semble désigner à la fois des fins substantielles, comme l'égalité sociale, et des procédures légitimes, comme la désignation par l'élection; mais elle semble ne pas pouvoir le faire en même temps et rendre ces deux exigences compatibles.

De là deux conceptions déterminées de la démocratie entre lesquelles il semble qu'il faille choisir.

La conception procédurale de la démocratie privilégie l'égalité dans la participation alors que la conception substantielle de la démocratie défend l'idée que celle-ci est un régime qui s'organise en vue de fins déterminées. L'une et l'autre se définissent largement dans leur opposition réciproque. La conception substantielle de la démocratie reproche à la définition procédurale sa duplicité: car restreindre la démocratie à un ensemble de règles, c'est faire semblant de ne pas voir qu'une démocratie implique des choix de valeurs sur des questions substantielles, donc qu'on ne peut pas simplement définir le régime démocratique par la forme des décisions prises. La conception procédurale de la démocratie reproche à cette définition substantielle son incohérence: à privilégier le contenu des décisions politiques, on finit par considérer que l'essentiel n'est pas dans l'implication de tous et qu'en toute rigueur une politique démocratique pourrait être menée par un pouvoir aristocratique ou oligarchique. Et considérer que la démocratie doit faire triompher une conception du bien, ce n'est pas faire droit au pluralisme, qu'on associe pourtant étroitement à la conception qu'on se fait d'une société démocratique, ouverte et tolérante.

Duplicité ou incohérence: ces deux arguments, remarquons-le, ne sont pas symétriques. Le premier entend montrer qu'on ne peut dissocier la forme de la décision de son contenu, sous peine de réduire la démocratie à n'être justement qu'une pure forme, sans réelle prise sur la sphère sociale; alors que le second montre que ne pas les séparer conduit à des conséquences non démocratiques. La démocratie substantielle reproche à la démocratie procédurale de ne pas avouer qu'elle est en réalité substantielle; la démocratie procédurale reproche à la démocratie substantielle de ne pas être réellement démocratique, c'est-à-dire de ne pas se définir par des procédures légitimes.

Je voudrais dans cet article réfléchir à la portée de ces critiques asymétriques et examiner plusieurs de ses interprétations. Je me demanderai en particulier comment on doit comprendre l'argument de la duplicité, dans la mesure où l'idée même de procédure, et avec elle l'idée d'une démocratie définie formellement, enveloppe plusieurs significations qu'il faut prendre soin de distinguer. Je m'interrogerai également sur le statut de l'égalité politique dans ces différentes façons de concevoir formellement la démocratie, 
et s'il est possible de la définir en termes exclusivement procéduraux. Plus précisément, je me demanderai si les conceptions de la démocratie procédurale, dans sa forme la plus répandue, ne doivent pas avouer que celle-ci n'existe qu'à partir d'une égalité qu'elle ne peut fonder par elle-même.

\section{Une invention procédurale de la loi?}

L'argument qui reproche à la démocratie substantielle sa duplicité est plus complexe qu'il en a l'air. L'idée générale sur laquelle il repose consiste à dire qu'il n'existe pas de démocratie formelle, puisque toute démocratie est dans son fondement substantielle. Mais cet argument demande explication. On peut, semble-t-il, l'interpréter de deux manières différentes.

La première interprétation consiste à dire qu'il n'est pas de politique sans décision et que toute décision est orientée par des valeurs, des interprétations, des représentations, bref qu'elle implique des choix substantiels. Il n'est pas, selon cette critique, d'État neutre: la procédure n'est nullement une garantie de neutralité, plus précisément celle-ci n'est qu'une apparence derrière laquelle se dissimulent des choix orientés. Nous avons sans doute là l'argument sur lequel se fondent toutes les critiques communautariennes du libéralisme rawlsien. Celui-ci se veut nettement procédural: il faut, selon Rawls, écarter les conceptions compréhensives du bien si l'on veut parvenir à un critère de justice qui puisse permettre la coexistence des doctrines morales ${ }^{1}$. Il faut pour cela parvenir à une interprétation politique de la justice, à travers la mise en place d'une "procédure de construction" dans laquelle «des agents rationnels représentant les citoyens et soumis à des conditions raisonnables, sélectionnent les principes destinés à gouverner la structure de base de la société ${ }^{2}$ ». Les communautariens objecteront qu'une communauté, quelle qu'elle soit, se définit par une conception substantielle de la vie bonne, et que c'est en fonction de ce critère que les préférences des uns et des autres peuvent être évaluées ${ }^{3}$.

Nous pouvons cependant considérer que l'argument de la duplicité est ici de faible intensité. Une conception procédurale de la démocratie ne met pas en question l'idée que les décisions sont motivées par des doctrines substantielles, mais entend au contraire faire en sorte que celles-ci puissent s'exprimer. Le problème est bien d'organiser, comme le souligne Rawls, la diversité des conceptions substantielles et non de la nier ${ }^{4}$. On peut penser que le constructivisme procédural ne parvient pas à organiser cette coexistence. C'est par exemple ce que Walzer reproche à Rawls: la position originelle et le contrat hypothétique proposés par Rawls échouent, parce qu'ils

1. J. Rawls, Libéralisme politique, trad. C. Audard, Paris, PUF, I995, p. 35 et suiv.

2. Ibid., p. I 27.

3. Voir sur cette critique W. Kymlicka, Les théories de la justice. Une introduction, trad. M. Saint-Upéry, Paris, La Découverte, 2003, p. 225 et suiv.

4. Rawls, Libéralisme politique, op. cit., p. I4. 
font abstraction des représentations partagées à partir desquelles il faut justement organiser l'espace démocratique ${ }^{5}$. Mais, à supposer que cette critique soit justifiée, elle ne revient pas à accuser le procéduralisme d'être en réalité hypocrite.

Mais il est une autre manière de comprendre la duplicité de la démocratie procédurale et celle-ci me paraît d'intensité supérieure. Cette interprétation consiste à dire qu'une démocratie procédurale n'existe pas, parce qu'une démocratie ne peut pas se contenter d'être seulement un ensemble de règles de fonctionnement: elle doit en effet engendrer des individus qui correspondent à ses institutions, c'est-à-dire qui obéissent aux lois, voire qui puissent se reconnaître en elles. Cet argument, plus fort, considère qu'il n'est pas de politique sans anthropologie politique: une démocratie doit former ses citoyens.

Cet argument est plus difficile à comprendre parce qu'il est le plus souvent mêlé au précédent. On peut s'en convaincre en lisant les critiques que Castoriadis adresse aux conceptions procédurales de la démocratie, en particulier dans son article "La démocratie comme procédure et comme régime ${ }^{6}$. Ces critiques sont profondes, sans être cependant dénuées de toute équivocité. C'est la définition habermassienne de la démocratie que Castoriadis a ici en vue (c'est la seule qu'il cite explicitement, en faisant référence à l'article de Habermas, "Trois modèles normatifs de la démocratie $\left.{ }^{7}\right)$. Habermas dans cet article compare la définition libérale de la démocratie et sa conception républicaine. Dans le premier cas, la fonction de la démocratie consiste à organiser la société comme un système de relations structurées par l'économie de marché. Dans le second cas, la politique est considérée comme constitutive du processus de socialisation dans son ensemble. Si, dans la société libérale, les citoyens sont définis par leurs droits subjectifs, dans la conception républicaine en revanche, le statut de citoyen vient des libertés positives qui permettent la participation à la pratique politique et la constitution d'une communauté de sujets libres et égaux. Le troisième modèle de démocratie qu'Habermas veut opposer aux deux autres, s'appuie sur un "concept procédural de politique délibérative ${ }^{8} »$ : il consiste à instaurer des procédures de communication permettant à une volonté commune de se former.

Castoriadis ne critique pas directement la conception habermassienne de la démocratie, mais entend en exhumer le fondement: pour Habermas (et pour Rawls également selon Castoriadis), il faut une définition procédurale

5. M. Walzer, Sphères de justice. Une défense du pluralisme et de l'égalité, trad. P. Engel, Paris, Seuil, I997, p. I23.

6. C. Castoriadis, La montée de l'insignifiance, Paris, Seuil, I996, p. 22 I-24I.

7. J. Habermas, "Trois modèles de démocratie», dans L'intégration républicaine, trad. R. Rochlitz, Paris, Pluriel, 20I4, p. 36I-382, cité par Castoriadis p. 226.

8. Ibid., p. 372. 
de la démocratie parce que la considérer comme un régime, «indissociable d'une conception substantive des fins de l'institution politique ${ }^{9}$ ", c'est du coup avoir une conception déterminée du bonheur des citoyens - ce qui est tendanciellement, selon eux, totalitaire ${ }^{10}$.

Contre cette conception procédurale de la démocratie, Castoriadis écrit :

Il est [...] impossible d'imaginer une loi, sauf précisément les lois de procédure, et encore, qui ne tranche sur des questions substantives. Même l'interdit du meurtre ne va pas de soi - à preuve les multiples restrictions, exceptions, qualifications dont il est partout et toujours entouré. Il en va de même pour ce qui est de l' 'application » de ces lois, qu'il s'agisse du judiciaire ou de l'«exécutif». Le juge ne peut (et en tout cas, ne doit) jamais être un Paragraphenautomat, parce qu'il y a toujours des «vides de droit»(Rechtslücken), mais surtout parce qu'il y a toujours une question d'interprétation de la loi et, plus profondément, une question d'équitée ${ }^{11}$.

Dans ce passage, Castoriadis a deux objets qu'il importe de séparer: la conception de la loi d'une part, son application d'autre part. Castoriadis considère, à juste titre, que les deux supposent un certain nombre de décisions et que celles-ci mettent en œuvre des valeurs sur lesquelles la collectivité a à se prononcer. Mais s'agit-il dans les deux cas du même type de décision? Et en quoi sommes-nous bien là en présence d'un argument opposable à toute forme de démocratie procédurale? Ces questions méritent qu'on s'y arrête, car le raisonnement de Castoriadis n'est pas immédiatement clair.

La conception moderne de la démocratie s'organise autour de l'idée que l'application de la loi n'est pas du ressort du peuple souverain, à qui il revient seulement de décider de son contenu. Mais Castoriadis voit là une illusion: celle selon laquelle il pourrait exister un régime purement exécutif dans l'application de la loi, qui ne serait pas alors confiée à un gouvernement ayant un pouvoir d'interprétation, mais à une administration. C'est à ses yeux un argument dirigé contre Habermas, puisque, refusant d'introduire des valeurs substantielles dans l'organisation de l'État, celui-ci est forcé de réduire le gouvernement à un pur organe d'exécution.

Cette interprétation de la pensée politique d'Habermas semble justifiée. V. Descombes, qui partage en la matière les mêmes convictions que Castoriadis, donne des arguments qui l'étayent. La conception qu'Habermas se fait de la démocratie conduit selon lui à ne maintenir que deux pouvoirs, le pouvoir législatif et le pouvoir administratif. V. Descombes cite notamment un passage de Droit et démocratie, dans lequel Habermas explique que dans l'État de droit, conçu dans les termes d'une théorie de la discus-

9. Castoriadis, «La démocratie comme procédure et comme régime», art. cit., p. 22I.

10. Ibid., p. 226.

11. Ibid., p. 230. 
sion, la souveraineté se retire dans les cycles communicationnels des débats publics, et la volonté des citoyens est alors liée au pouvoir administratif de l'appareil d'État ${ }^{12}$.

Il est sans doute nécessaire de défendre, contre l'idée d'un pouvoir exécutif purement administratif, la nécessité que revienne au gouvernement une part de décision. Mais rien ne permet de lier cette critique à la conception procédurale de la démocratie. Nous pouvons penser en effet qu'il n'y a pas de rapport nécessaire entre la définition habermassienne du modèle démocratique et l'écrasement de la fonction gouvernementale (ce que V. Descombes appelle «l'illusion nomocratique ${ }^{13}$ »). La démocratie procédurale que défend Habermas s'oppose au modèle libéral (qui met les opinions en concurrence) et au modèle républicain (qui recherche l'entente entre les citoyens). Mais n'est-ce pas dans ce dernier modèle, pleinement substantiel, qu'il faut combattre toute forme d'interprétation dans l'exécution de la loi au nom de la souveraineté des citoyens unanimes? Ne sont-ce pas les défenseurs du régime républicain qui considèrent qu'il n'est de décision politique qu'émanant des citoyens en corps, et qui ne voient dans la puissance exécutive qu'un organe délégué ? Nous devons à Rousseau d'avoir attiré notre attention sur cette question: la modernisation de l'idée républicaine suppose l'inaliénabilité de la souveraineté qui ne peut appartenir qu'au peuple en tant qu'il légifère. La liberté politique exige qu'on contraigne le gouvernement à n'être qu'un organe exécutif, un corps subordonné chargé d'appliquer les décisions publiques ${ }^{14}$. Voilà pourquoi

12. Ce passage est le suivant: «Dans l'État de droit conçu dans les termes d'une théorie de la discussion, la souveraineté du peuple ne s'incarne plus dans une assemblée concrètement identifiable de citoyens autonomes. Elle se retire dans les cycles communicationnels, pour ainsi dire sans sujets, des débats publics et des organismes. Ce n'est que sous cette forme anonyme que le pouvoir rendu fluide par la communication peut lier le pouvoir administratif de l'appareil de l'État à la volonté des citoyens" (Droit et démocratie, trad. R. Rochlitz et C. Bouchindhomme, Paris, Gallimard, I997, p. I 54). Voir V. Descombes, "Le contrat social de Jürgen Habermas", dans Le raisonnement de l'ours, Paris, Seuil, 2007, p. 35 I. V. Descombes cite également et commente l'argument de Castoriadis contre la conception procédurale de la démocratie dans Le complément de sujet, Paris, Gallimard, 2004, p. 392.

13. On ne peut, selon V. Descombes, remplacer le gouvernement par le pouvoir des lois (ou nomocratie), parce que la politique traite de problèmes pratiques, qui ne sont pas seulement des cas où une règle s'applique, mais le point d'application de plusieurs règles entre lesquelles la phronesis doit trancher. Voir V. Descombes, "L'illusion nomocratique ", dans Le raisonnement de l'ours, ibid., p. 287-309.

14. «Les membres [du gouvernement] s'appellent magistrats ou rois, c'est-à-dire gouverneurs, et le corps entier porte le nom de prince. Ainsi ceux qui prétendent que l'acte par lequel un peuple se soumet à des chefs n'est point un contrat ont grande raison. Ce n'est absolument qu'une commission, un emploi, dans lequel, simples officiers du souverain, ils exercent en son nom le pouvoir dont il les a faits dépositaires, et qu'il peut limiter, modifier et reprendre quand il lui plaît. L'aliénation d'un tel droit, étant incompatible avec la nature du corps social, est contraire au but de l'association ", Du contrat social, III, I, Paris, GF-Flammarion, 200I, p. 96 . 
il ne faut laisser au gouvernement aucune part dans l'interprétation de la volonté générale, explique Rousseau: ce serait favoriser sa tendance naturelle à usurper la souveraineté15. On peut sans doute considérer que ce projet est illusoire et que l'exécution de la loi suppose, a minima, une interprétation de ce qu'elle signifie, quand elle n'exige pas sa reformulation. Mais là n'est pas la question: car ce qui frappe ici, c'est que le régime substantiel du bien commun se pense comme nomocratie - preuve que l'écrasement de la fonction gouvernementale n'est pas lié à une conception procédurale de la démocratie.

Il faut sans doute alors déplacer la critique pour la rendre plus pertinente: ce qui est en question, c'est l'impossibilité, selon C. Castoriadis et $\mathrm{V}$. Descombes, de penser l'invention de la loi en termes seulement procéduraux. Mais sur ce point encore, nous pouvons penser que le modèle normatif proposé par Habermas ne tombe pas dans ces reproches. La démocratie délibérative qu'il appelle de ses vœux entend s'établir à partir d'une procédure idéale de discussion et de décision dont rien ne dit qu'elle n'ait pas à trancher dans des questions substantielles, à partir de ce niveau supérieur d'intersubjectivité vers lequel, pour Habermas, nous devons désormais tendre. Nous pouvons alors penser que dans cette perspective la démocratie procédurale n'est qu'un processus de formation de la décision sur des questions substantielles.

L'argument de la duplicité, dans sa version faible, ne paraît donc pas concluant. Dans sa version faible tout au moins: la restriction est ici d'importance, parce qu'il semble que la critique de nature anthropologique soit beaucoup plus déterminante et qu'elle constitue, en réalité, ce qu'une défense de la démocratie comme régime reproche à l'idée de procédure. Ce qui est en question, c'est justement ce qui est au cœur de la conception habermassienne de la démocratie: le rôle qu'est censée y jouer l'intersubjectivité. L'objection de Castoriadis prend dans cette perspective toute sa force: la duplicité n'est pas dans le fait de considérer que la procédure permet d'éviter les questions substantielles, mais dans le fait de penser qu'une société peut être constituée par les relations intersubjectives entre les individus. Une démocratie, explique Castoriadis, n'existe pas sans paideia:

Supposons même qu'une démocratie, aussi complète, parfaite, etc., que l'on voudra, nous tombe du ciel: cette démocratie ne pourra pas continuer plus que quelques années si elle n'engendre pas des individus qui lui correspondent, et qui sont, d'abord et avant tout, capables de la faire fonctionner et de la reproduire. Il ne peut y avoir de société démocratique sans paideia démocratique ${ }^{16}$.

La défense de la démocratie procédurale repose ainsi sur deux erreurs. La première consiste à réduire le social à l'intersubjectif. Une société ne peut

15. Ibid., III, Iо.

16. Castoriadis, «La démocratie comme procédure et comme régime», art. cit., p. 233. 
pas exister sur la seule base de discussions et d'échanges d'arguments rationnels, il faut des idées et des croyances communes ${ }^{17}$. La seconde consiste à réduire le citoyen à l'individu, considérant qu'un être humain se socialise dans des liens choisis de coopération, alors que nous recevons notre socialisation de significations imaginaires collectives. La philosophie politique contemporaine, selon Castoriadis, est plongée dans l'illusion: celle qui consiste à définir l'individu sans la société, tout en demandant, avec duplicité, aux institutions de faire exister l'esprit social sans lequel une démocratie ne peut exister ${ }^{18}$.

Voilà ce qui condamne la démocratie habermassienne: elle présuppose l'existence d'individus-substance, atomes humains autonomes qui seraient capables de tenir à distance leurs valeurs afin d'en délibérer et de discuter de leur identité collective. Or, explique Castoriadis, ces individus n'existent pas: nous sommes constitués par ce qu'il appelle du social-historique, par des traditions et des fins substantielles. Et dans ces conditions, la démocratie procédurale ne peut engendrer les individus qui lui correspondent sans les arracher, de manière autoritaire et hétéronome, à ce qui les constitue.

\section{Deux formes de démocratie procédurale}

L'argument de la duplicité semble donc porter à condition de l'entendre comme l'impossibilité pour la démocratie procédurale d'être un régime qui forme ses citoyens. Mais cette critique atteint-elle toute forme de démocratie procédurale? Rien n'est moins sûr. L'opposition entre substance et procédure est peut-être, dans cette perspective, trompeuse: trop massive, elle empêche de voir qu'il existe différentes manières de concevoir les procédures démocratiques. La démocratie habermassienne se définit à travers la mise en place de procédures de délibération, permettant la formation de l'opinion. Mais on peut comprendre l'idée de procédure de manière plus restrictive, comme le font les théories démocratiques schumpetériennes: comme méthode de désignation des gouvernants. Ceux qui s'en font les défenseurs font-ils également preuve de duplicité? Rien n'est moins sûr.

Schumpeter défend une approche réaliste et descriptive de la démocratie, à l'opposé de toute conception normative de ce qu'elle devrait être. Il faut arracher la démocratie à la réflexion philosophique, si on veut selon lui répondre définitivement aux critiques que cette réflexion n'a pas manqué de susciter. La démocratie, pour le dire autrement, n'est pas ce régime du peuple souverain, comme les traditions contractualistes et utilitaristes l'ont conçue depuis le $\mathrm{XVIII}^{\mathrm{e}}$ siècle: elle est le nom que l'on donne à la compétition pour le pouvoir politique entre des individus qui s'offrent au choix des électeurs. Ce qui signifie que la démocratie est un mode de gouvernement qui n'enveloppe en elle-même aucune fin politique déterminée, ni aucune conception

17. V. Descombes, Le complément de sujet, op. cit., p. 392.

18. Castoriadis, «La démocratie comme procédure et comme régime», art. cit., p. 223. 
du bien commun. Elle n'est, comme l'explique Schumpeter dans Capitalisme, socialisme et démocratie, qu'un «système institutionnel aboutissant à des décisions politiques, dans lequel des individus acquièrent le pouvoir de statuer sur ces décisions à l'issue d'une lutte concurrentielle portant sur les votes du peuple ${ }^{19}$ ». La démocratie est bien ainsi une méthode, qui est moralement indéterminée, en conséquence compatible avec n'importe quel système économique, que celui-ci soit capitaliste ou socialiste. C'est une pure procédure, neutre sur le plan des valeurs.

Cette définition est connue; son influence sur la théorie politique de la démocratie dans la deuxième moitié $\mathrm{du} \mathrm{xx}^{\mathrm{e}}$ siècle est grande ${ }^{20}$. Elle exclut qu'on puisse reprocher à cette forme procédurale de démocratie sa duplicité.

D’une part, la neutralité morale de la méthode démocratique n'enveloppe pas l'idée que les décisions politiques soient détachées des valeurs substantielles qui animent les citoyens. On peut même penser que cette méthode, parce qu'elle est substantiellement indéterminée, permet d'être acceptée par tous et d'arbitrer entre ces valeurs. Schumpeter considère en effet que sa théorie règle la question du rapport entre démocratie et liberté individuelle. La démocratie comme méthode ne garantit pas une liberté absolue (aucune forme de démocratie, selon Schumpeter, ne le fait), mais on peut estimer que, si chacun est libre de briguer le commandement politique en faisant acte de candidature électorale, la liberté d'opinion et de discussion sera large. Pour le dire autrement, la méthode démocratique organise un espace de confrontation pacifique des valeurs substantielles, par le jeu électoral de la désignation.

D'autre part, cette définition minimaliste semble pouvoir échapper à l'objection qui lie indissociablement démocratie et paideia. Le point de vue réaliste revendiqué par Schumpeter consiste précisément à définir la démocratie à partir des capacités actuelles des individus, et non à travers un sens politique qu'on pense qu'ils acquerront par la formation de leurs lumières et/ou par la pratique démocratique elle-même. La définition philosophique de la démocratie (la réalisation du bien commun par un peuple souverain), que Schumpeter entend définitivement écarter, ne repose selon lui sur aucun fondement: lorsqu'on dépasse la sphère des affaires privées, les individus n'ont qu'un sens affaibli des réalités et sont incapables d'avoir des volitions effectives. Ils généralisent avec peine, et s'ils peuvent manifester une compétence pour les affaires locales, au-delà ils perdent très vite le sens des réalités. Et, incapables de jugement politique, ils restent soumis aux préjugés et aux

19. J. Schumpeter, Capitalisme, socialisme et démocratie, (I942), trad. G. Fain, Paris, Payot, I990, p. 355 .

20. Sur cette influence, voir Carole Pateman, Participation and Democratic Theory, Cambridge, Cambridge University Press, I970, p. 5 et suiv. 
impulsions irrationnels ${ }^{21}$. Il faut donc proposer un mode de gouvernement qui soit en accord avec ce qu'ils sont. L'argument de la duplicité, si on comprend bien, est renversé: les partisans d'une démocratie substantielle définissent ce régime de telle sorte que la démocratie procédurale apparaisse comme illusoire, mais cette définition est vide si nul sujet ne lui correspond. Et c'est le cas, parce que la démocratie définie en termes substantiels (la recherche collective de l'intérêt général) est incapable de donner une conception précise de ce qu'est le bien commun, et du coup de déterminer la manière dont on accède à sa compréhension ${ }^{22}$. La conception substantielle de la démocratie définit un régime en quelque sorte suspendu, en attente de ce qu'il peut être, et de ce qu'il ne sera en réalité jamais.

Mais il faut aller plus loin encore: la neutralité substantielle de la démocratie correctement conçue, c'est-à-dire définie minimalement, doit nous empêcher de considérer comme antidémocratique les restrictions qu'il est nécessaire d'apporter dans la définition du demos. C'est aux yeux de Schumpeter une réalité historique: chaque démocratie définit sa base citoyenne ou électorale. Et certaines de ces restrictions sont des discriminations, qu'il faut condamner en tant que telles, mais non parce qu'elles seraient contraires à la nature de la démocratie. Schumpeter donne l'exemple de nations racistes qui associent aptitudes électorales et considérations d'ordre racial. Pour lutter contre ces discriminations, rien ne sert de dire qu'elles sont antidémocratiques, car elles ne le sont pas. Il faut laisser à chaque peuple le soin de se définir lui-même - c'est une condition essentielle de la méthode démocratique. Il faut combattre ces doctrines en ellesmêmes, en montrant qu'elles sont fausses ou injustes - les critiquer d'un point de vue moral et non politique, extrinsèque et non intrinsèque ${ }^{23}$. Pour le dire autrement, l'idée de démocratie n'enveloppe pour Schumpeter aucune valeur spécifique.

On peut sans doute considérer dans cette perspective que la définition réaliste de la démocratie, comme méthode de désignation, est la plus inclusive qui soit, puisqu'elle ne présuppose aucune compétence politique spécifique. Mais ce n'est pas là une raison pour définir la démocratie de cette manière: l'égalité politique n'est nullement une condition substantielle de ce qui n'est qu'une méthode de désignation.

Cette conclusion heurte nos convictions concernant la démocratie. Car nous pensons qu'elle est le nom, de manière générale, que l'on donne au régime de l'égalité politique. Mais sur quel argument fonder cette conviction? Nous avons là un problème de point de vue. Considérer qu'il n'y a pas de démocratie sans égalité politique, n'est-ce pas introduire un point de vue substantiel et normatif pour juger d'une conception de la démocratie qui

21. J. Schumpeter, Capitalisme, socialisme et démocratie, op. cit., p. 346 et suiv.

22. Ibid., p. 330-33I.

23. Ibid., p. 323. 
entend précisément s'affranchir de ces critères? D’un point de vue procédural, cette objection peut sembler très extérieure. Nous sommes ainsi renvoyés à ce face-à-face entre procéduralisme et substantialisme, définis dans leur opposition respective.

L'égalité politique a cependant une justification procédurale, qu'on peut énoncer de l'intérieur même du schumpetèrianisme. On trouve par exemple chez Robert Dahl un tel argument. R. Dahl défend une conception formelle de la démocratie, dont les principes sont ceux de l'auteur de Capitalisme, socialisme et démocratie: il rejette les modèles normatifs (qui sont à ses yeux purement idéaux), il définit la démocratie (qu'il appelle polyarchie) comme un système institutionnel de désignation (et non comme l'expression de la volonté générale). Il existe, selon lui, deux méthodes pour définir la démocratie: la maximisation et la description. La première consiste à fixer une fin politique et à concevoir la démocratie de telle sorte qu'elle permette d'atteindre cette fin. La seconde consiste à décrire les régimes qu'on nomme démocratie, afin de saisir leurs traits communs ${ }^{24}$. La maximisation est à rejeter, parce qu'elle est utopique: elle pousse à condamner tous les régimes existants, puisqu'aucun ne permet d'atteindre cette fin politique, quelle que soit sa nature, fixée a priori. La description permet de fixer les conditions minimales de la démocratie: des élections, et une compétition politique entre citoyens ou partis. Une démocratie se définit par un ensemble de procédures, et non par des fins substantielles ${ }^{25}$.

Schumpetérien, R. Dahl ne l'est pourtant pas jusqu'au bout, notamment à propos de l'égalité politique. Son argument (qu'on trouve exposé dans un article publié en I979) est le suivant ${ }^{26}$ : l'égalité politique ne peut pas être fondée sur l'idée d'une compétence politique, car un tel fondement est démenti par les faits. Nous savons bien que le demos ne peut pas comprendre l'ensemble de la population et qu'il faut bien par exemple que l'on s'accorde sur une majorité civile. Mais ce n'est pas une raison pour penser que l'inclusion est contingente. Car la méthode démocratique suppose l'égalité politique afin que la procédure de désignation puisse paraître légitime et donc conduire à ce qu'elle est censée produire: une sélection électorale non contestée. La procédure démocratique implique la participation pour conduire à un résultat légitime.

Autant dire que R. Dahl borde, ou s'efforce de border, la conception schumpetérienne de la démocratie en donnant à l'égalité politique une justification procédurale, c'est-à-dire non fondée sur une égalité substantielle

24. R. Dahl, Preface to Democratic Theory, rééd. Chicago, The University of Chicago Press, 2006, p. 63 et suiv.

25. Sur l'évolution de R. Dahl et sur sa conception procédurale de la démocratie, voir Richard N. Krouse, "The Changing Theory of Robert Dahl », Polity, vol I4, n 3 (Spring I982), p. $44 \mathrm{I}-463$.

26. "Procedural Democracy», dans Philosophy, Politics and Society, Peter Laslett et James Fishkin (dir.), Fifth Series, I979, p. 97-I33. 
antécédente dont cette égalité politique serait une expression. Il la rend en quelque sorte plus cohérente: si la démocratie est une opération de désignation, cette procédure doit pouvoir mener à un résultat non contesté, sous peine de ne pas être opératoire. Pour le dire autrement, R. Dahl justifie l'égalité politique de manière intrinsèque, donc sans faire appel à des valeurs substantielles.

\section{Égalité politique, politique de l'égalité}

Il semblerait donc possible de donner un fondement procédural à l'égalité politique, donc de montrer que la démocratie procédurale peut fonctionner sans se donner en sous-main une assise substantielle cachée. Mais cette justification est-elle convaincante? Elle se heurte selon moi à deux objections. D'abord, nous pouvons penser que cet argument est d'ordre prudentiel et non stricto sensu nécessaire: il est important que l'inclusion soit la plus grande possible afin que le résultat de la procédure soit accepté, mais de là on peut difficilement inférer que l'idée d'égalité politique est analytiquement contenue dans l'idée de procédure démocratique. Ensuite, et peut-être plus profondément, quand bien même procédure et égalité politique seraient indissociables, il peut sembler que la démocratie demande bien davantage pour ce qui est de l'égalisation. Ou plus précisément, il peut sembler que l'égalité politique ait besoin pour exister que la démocratie s'organise comme le régime général de l'égalité. L’argument est classique, mais il n'est pas pour autant dépourvu de pertinence. Il revient à dire que les droits politiques sont formels s'ils ne sont pas accompagnés de droits économiques et sociaux (argument formulé par Marx dans La question juive mais dont on peut trouver des avant-courriers dans l'égalitarisme de Rousseau ${ }^{27}$ ). Thomas Nagel en donne une formulation moderne plus précise: l'égalité politique, juridique et sociale ne peut être garantie seulement par elle-même, il faut qu'elle soit fondée sur une égalisation des ressources. Le droit de vote et celui d'occuper une charge publique est une condition nécessaire de l'égalité politique, tout comme le droit d'être juré et de porter plainte celle de l'égalité juridique, et l'abolition des titres et des privilèges celle de l'égalité sociale. Mais ces conditions sont insuffisantes en elles-mêmes: "D'importantes et considérables inégalités en pouvoir politique, protection juridique, considération sociale et respect de soi sont compatibles avec ces conditions formelles ", explique-t-il dans Questions mortelles ${ }^{28}$. Pour le dire autrement, l'égalité politique suppose bien plus que des droits politiques. Nous voilà alors amenés à reformuler l'argument de la duplicité: une démocratie procédurale ne peut exister sans égalité politique, mais celle-ci suppose que la démocratie mette en œuvre une politique de l'égalité.

27. Voir Rousseau, Du contrat social, II, I I.

28. Thomas Nagel, Questions mortelles, trad. P. Engel et C. Engel-Thiercelin, Paris, PUF, 1983 , p. I28. 
Cette objection est-elle recevable? Je veux dire: l'est-elle du point de vue de la démocratie procédurale? Est-ce que ses défenseurs, face à cet argument, doivent avouer que la méthode démocratique ne peut pas fonctionner si l'égalité n'est pas la fin de la démocratie — si la démocratie ne devient pas un régime, comme l'écrit Castoriadis? Ce n'est pas si simple, car on peut rétorquer qu'il n'est pas nécessaire que cette égalité politique soit pleine et entière, qu'il suffit que le droit de vote assure une égalité arithmétique générale qui soit la plus inclusive possible, bref que l'égalité formelle suffit très largement pour que la méthode démocratique fonctionne, dans la mesure où on attend d'elle qu'elle désigne des gouvernants.

Mais est-ce bien le cas? Est-ce que les droits politiques formels construisent suffisamment ce sentiment d'égalité qui permet à la méthode démocratique de fonctionner pleinement comme procédure de désignation? Le problème est qu'on peine à voir ici comment ce sentiment se construit, c'est-à-dire ce qui donne à chaque individu la conscience d'être inclus dans une communauté. Le droit de vote nous met tous sur un pied d'égalité, mais nous savons bien que, dans les démocraties de masse, notre voix est insignifiante. C'est l'argument de Dworkin: l'égalité politique est faiblement réalisée lorsqu'elle prend la forme d'une égalité d'impact, définie comme capacité à avoir tous le même effet sur le processus politique. Selon cette conception de l'égalité politique, "l'opinion que chacun finit par former dans le processus bénéficiera d'un poids égal dans la décision finale de la communauté ${ }^{29}$ ». Mais s'il est vrai que, par le droit de vote, chaque individu appartenant au collège électoral détient le même pouvoir d'agir sur les politiques publiques que les autres, cette égalité n'a pas de sens dans des communautés politiques où les individus sont trop nombreux pour considérer qu'ils ont un pouvoir réel. De même, l'égalité d'influence sur les discussions politiques est imparfaite. Elle se définit comme répartition égale du pouvoir politique, de telle sorte que chaque opinion a les mêmes chances d'avoir une expression politique, donc les mêmes chances de persuader d'autres individus. Cette égalité n'existe pas en réalité: certains individus sont plus influents que d'autres, et c'est d'ailleurs souhaitable. Nous ne pouvons pas regretter, explique Dworkin, que Martin Luther King ait eu davantage de poids que n'importe quel citoyen dans le combat qu'il a mené30.

Faut-il alors considérer que l'égalité politique n'est qu'une chimère? Non, parce qu'il existe une troisième forme d'égalité politique, ou une troisième manière d'interpréter l'égalité politique: c'est l'égalité dans l'attention que la communauté politique porte aux individus. Cette égalité d'attention, que Dworkin privilégie, est une égalité de statut politique: la démocratie est le régime qui considère également les individus.

29. R. Dworkin, Justice pour les hérissons, trad. J. Jackson, Genève, Labor et Fides, 2015 , p. 4I9.

30. Ibid., p. 420. 
L'égalité arithmétique d'impact est procédurale. Elle n'a donc pas de valeur en elle-même. Dworkin ne nie pas son importance, mais ne voit pour elle aucune justification intrinsèque: "Si la légitimité d'un arrangement politique pouvait être améliorée par des arrangements constitutionnels qui créent une certaine inégalité d'impact mais ne comporte aucune nuance ni aucun danger d'indignité, il serait pervers d'exclure de telles mesures $^{31}$. » L'anti-égalitarisme d'un point de vue procédural peut servir l'égalité politique réelle dont la démocratie a besoin. L'hypothèse n'est pas à exclure, car des cas de cet ordre peuvent se présenter. Dworkin ne donne pas d'exemples explicites, mais certains se présentent à l'esprit. Ainsi, nous pouvons penser qu'un découpage électoral peut légitimement donner plus d'impact à certaines populations défavorisées, afin de compenser une inégalité d'attention.

Cette argumentation n'est pas sans faille. D'abord, elle peut être suspectée d'incohérence. Il est étrange, peut-on en effet objecter, de séparer l'égalité d'attention et l'égalité d'impact, puisque, sauf à tomber dans une forme de paternalisme difficilement justifiable, on peut considérer que l'égalité dans la participation est la première forme de l'égalité d'attention de la communauté politique. Ensuite, il peut sembler difficile de mesurer l'égalité d'attention: dans quelle mesure chacun pourra-t-il être convaincu qu'il est l'objet d'une sollicitude égale ? Quel type de résultats économiques et sociaux faut-il obtenir, par la redistribution, pour parvenir à une telle conviction?

Ces objections sont sans doute recevables, mais là n'est pas l'essentiel. Ce que montre Dworkin, ou plus justement ce qu'il nous permet de penser, c'est que la question de l'égalité politique ne peut pas être considérée sans qu'on prête attention au sentiment d'inclusion auquel la démocratie doit donner lieu, et qui est aussi sa condition d'existence. Le tort de la démocratie procédurale n'est pas de négliger ce sentiment d'inclusion, mais de considérer qu'il peut être produit par la seule participation à la méthode qui consiste à désigner les gouvernants. Les défenseurs de la procédure raisonnent mal: ils inversent les causes et les effets. Car le vote ne produit pas l'égalité, mais en est un effet - et, de même, l'intersubjectivité est un effet et non une cause de l'inclusion.

Il n'y a donc de démocratie qu'à partir d'une égalité antécédente, à partir d'une communauté des égaux qui ne peut advenir que parce que les individus se considèrent substantiellement comme des égaux. Mais cette égalité politique et sociale substantielle, Castoriadis a raison de souligner qu'elle ne peut se déduire d'une égalité non politique antérieure, naturelle ou religieuse $^{32}$. Nous ne naissons pas égaux, nous proclamons que nous nais-

31. Ibid., p. 423 .

32. C. Castoriadis, "Nature et valeur de l'égalité", dans Domaines de l'homme, Paris, Seuil, I986, p. 3I7. 
sons égaux, et nous construisons cette égalité sociale de telle sorte que tous se sentent inclus dans une communauté. L'égalité doit être la fin de la démocratie, parce que justement elle en est la condition. La démocratie pose l'égalité comme valeur sociale et comme objet des politiques publiques. Ce qui suppose qu'on travaille à la rendre compatible avec les valeurs caractéristiques d'une société ouverte (la liberté, l'efficacité). Et parce que la démocratie fait naître l'égalité dont elle dépend, il faut bien une padeia pour former les individus à l'égalité, pour qu'ils ne cessent de se considérer comme des égaux. La démocratie est substantielle non pas parce qu'elle est un régime proposant un genre de vie, mais parce qu'elle est le régime de l'égalité.

\section{Conclusion}

Les critiques de la démocratie procédurale dénoncent ce qu'elles considèrent comme une duplicité: elle reposerait sur des fondements substantiels qu'elle dissimule et/ou se dissimule. Cet argument n'est que partiellement vrai. En toute rigueur, il ne porte que sur les procédures de délibération et non sur les procédures de désignation. La délibération ne peut exister que s'il existe une communauté politique qui se construit à partir des convictions partagées, à partir de valeurs substantielles dont l'éducation nous permet de discuter. Ce qui signifie sans doute qu'on ne peut définir la démocratie comme seulement délibérative. Mais cet argument n'empêche pas d'en donner une définition minimale. Les conceptions schumpetériennes de la démocratie sont plus rigoureuses dans cette perspective que sa définition habermassienne.

Mais si elles sont en quelque sorte moins hypocrites parce qu'elles ne font pas semblant de se passer d'un fondement substantiel, elles restent incohérentes. Car, quoi qu'on fasse, la démocratie procédurale de désignation ne peut être elle-même qu'à partir de conditions substantielles. Encore faut-il bien comprendre ce qu'on désigne ici comme valeurs substantielles. Il faut en distinguer deux sortes: les doctrines compréhensives (morales ou religieuses) et la doctrine démocratique de l'égalité. Elles ne sont pas à mettre sur le même plan. Les premières sont des convictions à partir desquelles une démocratie ne peut se définir sans mettre grandement en question la tolérance et le pluralisme qui la caractérise à l'âge contemporain. La doctrine de l'égalité est, elle, fondamentale: la démocratie ne peut pas tenir ses promesses si elle n'est pas une communauté où chacun se considère comme également traité. Même définie minimalement, la démocratie appelle cette doctrine de l'égalité. Les partisans d'une telle conception doivent admettre que les procédures démocratiques ne peuvent sembler légitimes que si l'égalité politique est réelle. La définition procédurale de la démocratie, pour être cohérente, exige une attention publique à l'égalisation.

On ne peut donc pas reprocher à la démocratie procédurale minimaliste sa duplicité, mais on peut lui reprocher son incohérence: la démocratie 
de désignation souligne l'importance de l'égalité, mais refuse que celle-ci fasse l'objet d'une politique substantielle. Elle considère que l'égalité est la condition de la démocratie sans voir que, pour qu'elle en soit la condition, il faut aussi qu'elle en soit la fin. 